\title{
Direct observation and analysis of crack front morphology during crack pinning by heterogeneous interface
}

\author{
D. Dalmas ${ }^{1, a}$, E. Barthel ${ }^{1}$ and D. Vandembroucq ${ }^{2}$ \\ ${ }^{1}$ Laboratoire "Surface du Verre et Interfaces" (SVI) - unité mixte CNRS/Saint- \\ Gobain, Aubervilliers, France \\ ${ }^{2}$ Laboratoire PMMH, Paris, France
}

\begin{abstract}
Adhesion of thin film multilayers deposited on glass is a crucial issue for many industrial applications. Thus, it becomes of great interest to measure and also to increase the adhesion. Many mechanisms of toughening a brittle solid can be found in literature; but few of them can be applied to thin film layer. By introducing a heterogeneous interfacial toughness field, it should be possible to increase adhesion. This toughness modification would be the consequence of the existence of a pinning regime due to a local change of the toughness. To experimentally validate this new approach of adhesion modification, we investigate the crack front pinning by performing cleavage tests on multilayer coated samples with a heterogeneous interfacial toughness. We have tested different patterns of pinning region. The crack front morphology was nicely described in the framework of the perturbative approach initially developed by Gao and Rice and allowed us to determine the local value of the energy release rate ( adhesion).
\end{abstract}

\section{INTRODUCTION}

Thin film multilayers deposited on glass are widely used for flat optical, photoelectric and electrochromic devices. In many applications, adhesion is a crucial issue. This is the case for instance when mechanical strength is required for further processing or for integration in complex systems. Thus, it becomes of great interest for those applications to increase the adhesion. Many mechanisms of toughening a brittle solid can be found in literature (local transformations, crack bridging, crack trapping, microcracking...); but few of them can be applied to thin film layer. One possible way to increase the adhesion of an interface between two brittle solids is to create a composite interface in order to modify the crack front morphology due to pinning on region of higher toughness. By a judicious choice of the heterogeneous interfacial toughness field, it could be possible to have an adhesion which will be higher than the simple mean value of the local toughness. This toughness modification would be the consequence of the existence of a strong pinning regime which is characterised by a rapid local change of the toughness in the direction of crack propagation. Crack pinning has been widely theoretically and experimentally studied since more than two

\footnotetext{
a e-mail : davy.dalmas@saint-gobain.com
}

This is an Open Access article distributed under the terms of the Creative Commons Attribution-Noncommercial License 3.0, which permits unrestricted use, distribution, and reproduction in any noncommercial medium, provided the original work is properly cited. 
decades, but few, if any, of these experimental studies have been done in the past in a manner which allows evaluating the front morphology modification due to pinning by a single defect.

Since the pioneering work of Rice [1] and subsequent developments by Gao and Rice [2], the study of crack front propagation in heterogeneous media has received an increasing interest [3-7]. Unfortunately, most of these works are theoretical; the lack of experimental studies to test the relevance of crack propagation models is due to the fact that in most cases the direct observation of a crack front during propagation is impossible. Moreover, using indirect techniques to localize the front position such as acoustic emission is not relevant because the spatial resolution is not high enough.

To experimentally validate this new approach of adhesion modification, we present an experimental study of purely interfacial crack propagation in the presence of very simple, macroscopic, and fully described heterogeneous toughness field. We will try to capture the individual contribution of each components of the toughness field by analyzing the crack front morphology in the framework of the crack pinning theory.

\section{EXPERIMENTS}

The set-up we have developed enables us to propagate and monitor planar crack in transparent samples with a patterned interface by using a DCB cleavage test (Double Cleavage Beam) $[8,9]$.

The main advantages of this set-up are:

- a direct observation by using a CCD camera of the crack front morphology is possible due to the transparent samples

- the crack is restricted to a strictly planar geometry because propagation occurs at the weakest interface of a thin multilayer stacking.

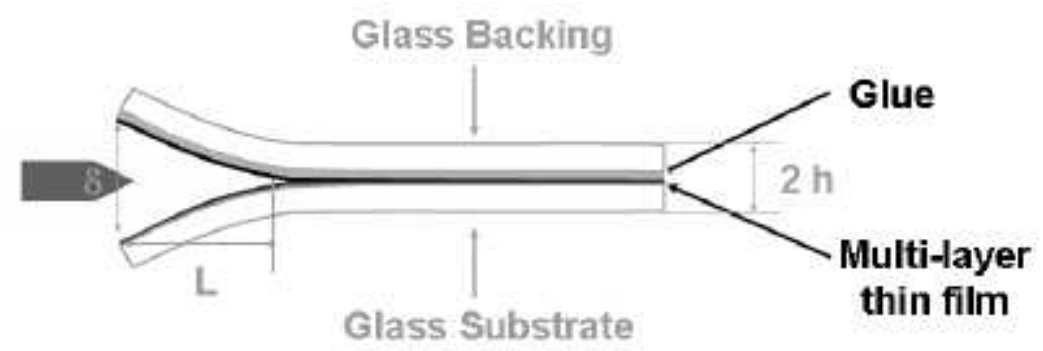

Fig. 1. Schematic view of the double cleavage beam test (DCB)

By scratching the multilayer coating we are able to introduce an heterogeneous toughness at the weakest interface of our DCB. The objective is to introduce a toughness pattern at this particular interface (i.e. a local replacement of the weak interface by an interface of stronger adhesion). Actually, we have shown that performing a scratch via a ball on plane tribometer leads to a removal of the coating down to the weak interface. Then, during the gluing of the glass backing, zones of strong adhesion are generated in the scratch traces (Fig. 2). 


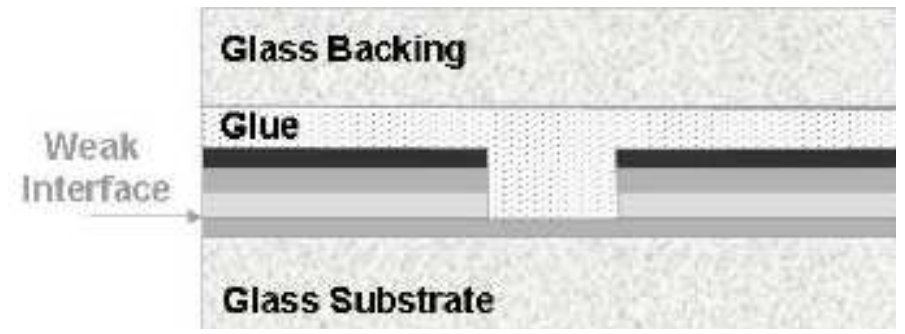

Fig. 2. Schematic view of the toughness patterning at the weakest interface of a multilayer coated glass substrate.

In this study, samples with different toughness patterns have been generated. Two examples are given on Fig. 3.
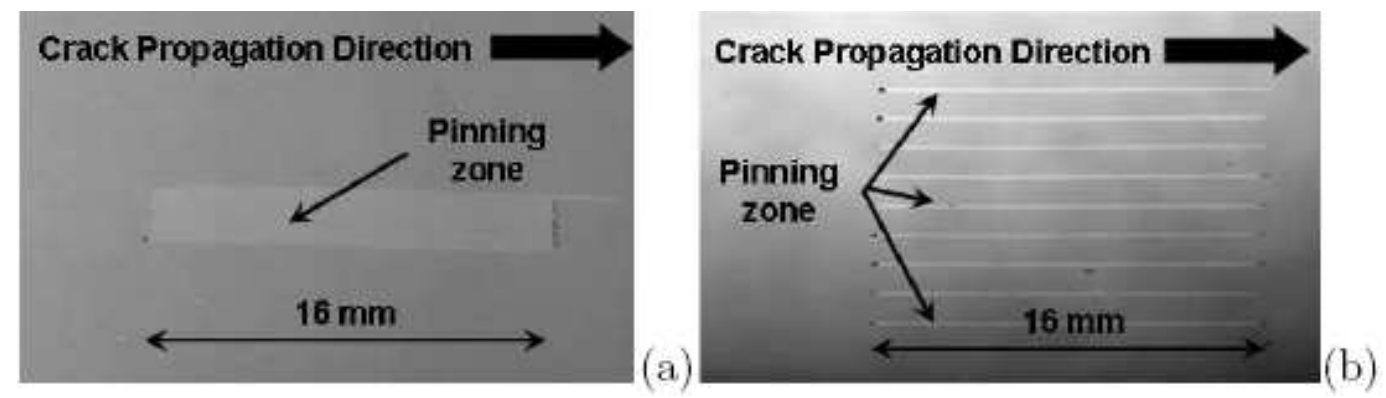

Fig. 3. Top view of the pinning zones of two samples.

\section{RESULTS: CRACK FRONT MORPHOLOGY EVOLUTION}

Fig. 4 gives the evolution of the crack front morphologies during cleavage tests. This evolution can be divided in four successive stages:

- $\quad$ Stage 1: Propagation of the front without any modification of the morphology as the front propagates in an homogeneous part of the DCB sample.

- Stage 2: Accommodation period of the front morphology. Due to the presence of the zone of higher toughness, the front morphology is locally modified.

- Stage 3 : weak pinning regime. During this stage, the motion of the front is a simple translation with a constant shape. This stage lasts until the front leaves the pinning zone.

- Stage 4: return of the front in the homogeneous zone of the sample. 

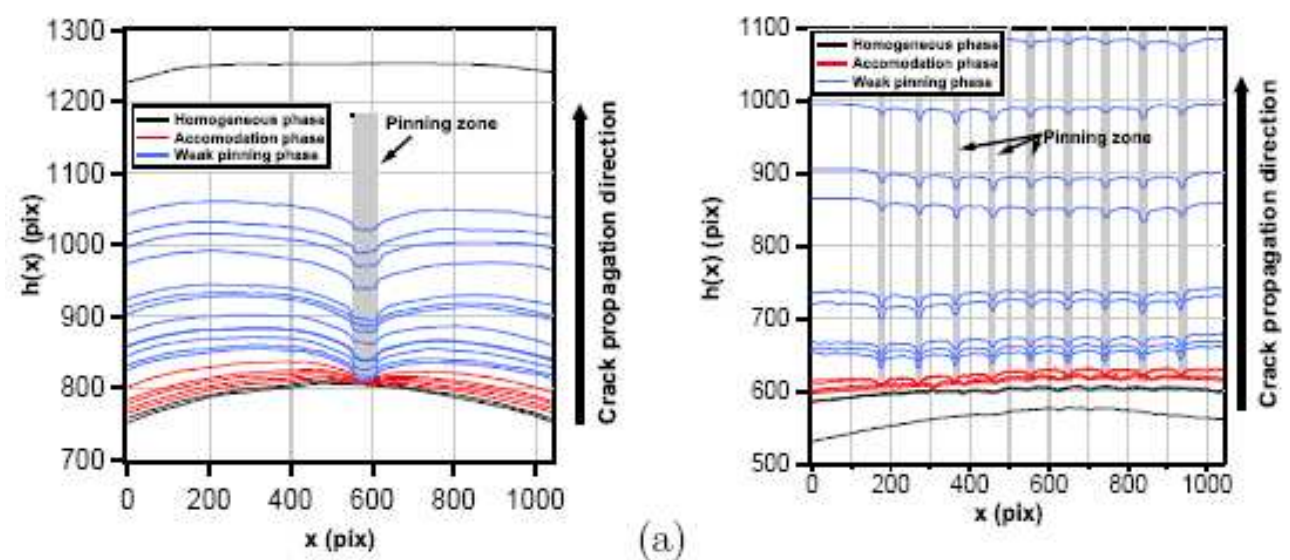

(a)

Fig. 4. Evolution of the front morphology during cleavage test for both the single line pinning zone (a) and for the network of parallel pinning zones (b).

\section{ANALYSES}

\subsection{Theoretical expression of front morphology in presence of a single pinning zone}

In order to see if the first order perturbative analysis of Gao and Rice [2] can be used to fit our measurements of crack front morphologies $\mathrm{h}(\mathrm{x}$ ) (cf. Fig. 5) we have to invert the following expression :

$$
K(x, h(x))=K_{0}\left(1+\frac{1}{2 \pi} \int \frac{h(x)-h\left(x^{\prime}\right)}{\left(x-x^{\prime}\right)^{2}} d x^{\prime}\right)
$$

Where $\mathrm{x}$ is crack propagation direction, $\mathrm{h}(\mathrm{x})$ the front roughness, $\mathrm{K} 0$ the macroscopic equivalent stress intensity factor.

By using a simple boxcar function $\mathrm{b}(\mathrm{x})$, it is possible to describe the toughness distribution $\mathrm{Kc}(\mathrm{x})$ in presence of a single pinning zone of constant toughness with width $2 \mathrm{a}$ as follows:

$$
K(x)=K_{r e f}\left(1+\frac{\Delta K}{K_{r e f}} b(x)\right)
$$

Where $\mathrm{K}_{\mathrm{ref}}$ is the toughness of the original material.

Then by using a Fourier transform of both previous equations, we are able to obtain a relation between $\mathrm{h}(\mathrm{x}), \mathrm{b}(\mathrm{x})$ and the toughness variation $\Delta \mathrm{K}$. This relation in Fourier space, which is a product of two functions, leads to the following expression in direct space:

$$
h(x)=\frac{1}{\pi^{2}} \frac{\Delta K}{K_{0}}\{\log |x| * b(x)\}
$$

Where * stands for the convolution product operator. 
This last direct expression of the front morphology $\mathrm{h}(\mathrm{x})$ was introduced in a routine developed in order to fit the experimental data. The adjustment parameters are the toughness contrast $(\Delta \mathrm{K} / \mathrm{K} 0)$ and the width of the pinning zone (a).

\subsection{Analysis of front morphologies}

A comparison between the experimental front morphology and the fit is given in Fig. 5 for some representative front positions for both samples (i.e. single line and pattern of parallel lines).
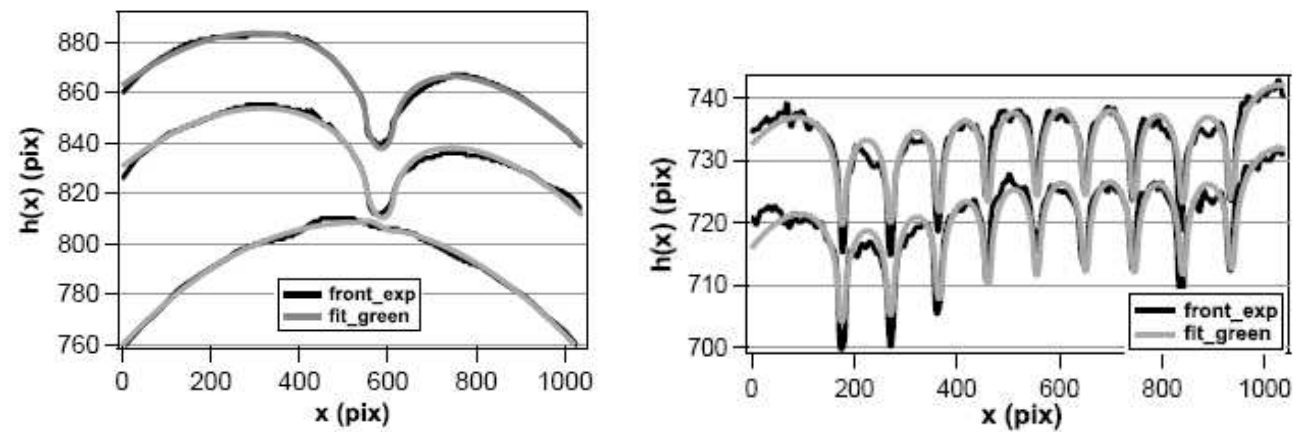

Fig. 5. Comparison of experimental measurements of front morphology (black lines) and fits (gray lines) obtained by using a first order perturbative analysis for both samples.

In all the parts of the sample, the description of the experimental curves by the analytical expression of equation 3 is of good quality. In the pinning region, the agreement between fits and experimental data demonstrates that it is possible to describe our front measurements in the framework of the pinning theory developed by Gao and Rice.

In Fig. 6, the evolution of the half width of the pinning zone and of the relative toughness contrast is plotted as a function of the front position for the single line pinning zone. These vales of these two parameters are coming from the fits which were performed on the experimental data. These obtained evolutions are for both parameters in correlation with the evolution of the front morphology which was described in section 3.

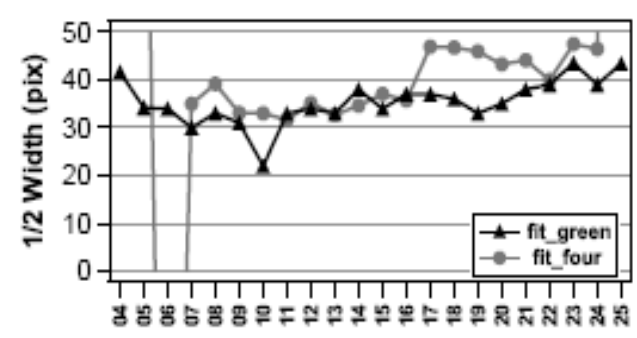

Front $\mathbf{n}^{\circ}$

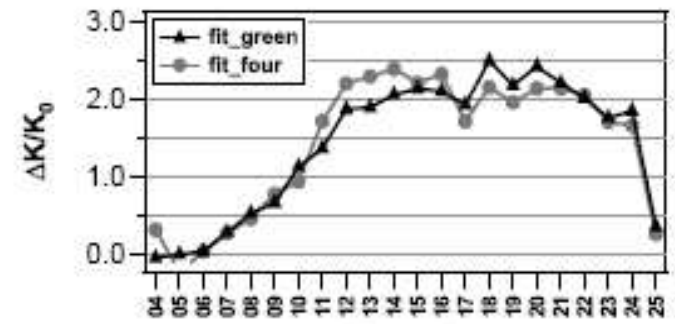

Front $\mathrm{n}^{\circ}$

Fig. 6. Evolution of the width of the pinning zone (left) and of the toughness variation (right) as a function of the position of the crack front for the a single wide pinning zone sample.

\section{CONCLUSION}

In this study, the propagation of a crack front along a patterned interface was studied experimentally. All the results show that our experimental set-up allows analysing the modification of a front by one- 
dimensional tough patterns within the framework of the pinning theory. The quantitative agreement obtained in this series of experiments between the measurements and the analytical predictions thus motivates us to apply the same strategy to more complex patterns.

\section{References}

1. J. R. Rice, First-order perturbation in elastic fields due to variation inlocation of a planar crack front. J. Appl. Mech. 52, 571-579 (1985)

2. Gao H., J. R. Rice, First-order perturbation analysis of crack trapping by arrays of obstacles. J. Appl. Mech. 56, 828-836 (1989)

3. K. J. Maloy, A. Hansen, E. L. Hinrichsen, S. Roux, Experimental measurements on the roughness of brittle cracks. Phys. Rev. Lett. 68, 213-215 (1992)

4. T. M. Mower, A. S. Argon, Experimental investigations of crack trapping in brittle heterogeneous solids. Mech. Mater. 19, 343-364 (1995)

5. D. Bonamy, Intermittency and roughening in the failure of brittle heterogeneous materials, J. Phys. D 42, 214014 (2009)

6. S. Ramanathan, D. Ertas, D. S. Fisher, Quasistatic crack propagation in heterogeneous media. Phys. Rev. Lett. 79, 873-876 (1997)

7. S. Roux, D. Vandembroucq, F. Hild, Effective toughness of heterogeneous brittle materials. Eur. J. Mech. A 22, 743-749 (2003)

8. E. Barthel, O. Kerjan, P. Nael, N. Nadaud, Asymmetric silver to oxide adhesion in multilayers deposited on glass by sputtering. Thin Sol. Films 473 (2), 272-277 (2005)

9. D. Dalmas, E. Barthel, D. Vandembroucq, Crack front pinning by design in planar heterogeneous interfaces, J. Mech. Phys. Solids 57, 446-457 (2009) 